\title{
Keratin Expression in
}

\section{Mammary Paget's Disease in situ with Intraductal Invasion}

\author{
Ichiro Kurokawa $^{a}$ Sayaka Furukawa ${ }^{a}$ \\ Masahiro Kishimoto $^{b}$ Ayako Kakuno $^{c}$ Airo Tsubura $^{d}$ \\ Departments of ${ }^{\mathrm{a}}$ Dermatology, ${ }^{\mathrm{b}}$ Breast Surgery and ${ }^{\mathrm{c}}$ Pathology, Meiwa \\ Hospital, Nishinomiya, and ${ }^{\mathrm{d}}$ Department of Pathology, Kansai Medical \\ University, Osaka, Japan
}

\section{Key Words}

Mammary Paget's disease $\cdot$ Keratins $\cdot$ Intraductal carcinoma

\begin{abstract}
We performed immunohistochemical studies of epithelial keratins in intraductal carcinoma in situ (IDCIS) in mammary Paget's disease (MPD). K7, K8 and K18 were expressed in IDCIS in MPD. However, K19 was not expressed in IDCIS in MPD. Interestingly, K17 was expressed in some tumor cells in IDCIS. K17, a hyperproliferative keratin, may suggest ductal invasion and poor prognosis in MPD.
\end{abstract}

\section{Introduction}

Mammary Paget's disease (MPD) is a rare skin carcinoma. It comprises $0.5 \%$ of all breast cancers. Clinically, it is difficult to distinguish MPD and chronic eczema. The diagnosis of MPD can be made by histopathological evaluation. According to the extent of tumor invasion, MPD is classified into two types: carcinoma in situ (Tis) and pagetoid carcinoma. We performed immunohistochemical studies of epithelial keratins in intraductal carcinoma in situ (IDCIS) in MPD.

\section{Case Presentation}

A 57-year-old Japanese woman first noticed erosion on the right nipple (fig. 1) 8 years ago. She was treated topically with corticosteroid cream by her practitioner. However, there was no clinical response. Therefore, skin biopsy was performed. Histopathological study revealed intraepidermal nests with Paget cells in the epidermis. A histopathological diagnosis was made of MPD. Mammography showed no demarcated tumor of the breast. Magnetic resonance imaging (MRI) 
showed thickened epidermis suggesting an intraductal invasion in the breast duct. Elastography showed sporadic firm areas in the nipple, areola and breast duct. The patient underwent radical mastectomy in the sentinel node biopsy. No metastasis was found in the sentinel nodes. Histopathological findings on the excised specimens showed intraductal invasion with Paget cells in breast duct.

\section{Discussion}

The origin of Paget cells is unclear. Toker cells, clear cells in the epidermis, are possible precursor cells of Paget cells [1]. Keratins are important markers in evaluating the origin of epithelial tumors, and are dependent on the stage of differentiation and prognosis of the carcinoma. In the normal mammary gland, luminal cells express K1, K8, K15, K16, K18 and K19, whereas basal (myoepithelial) cells express K5, K14 and K17 [2]. Toker cells, K7 positive, are observed in 10\% of men and women in the nipple. Keratin expression in intraductal invasion in MPD has not been reported. We performed immunohistochemical studies of epithelial keratins (K1, K7, K8, K10, K14, K15, K16, K17, K18, K19 and K20) in MPD. The results showed K7, K8, K18 and K19 were expressed in intraepidermal nests containing Paget cells (fig. $2 \mathrm{a}-\mathrm{d}$ ). Tumor cells were negative for K17. In the breast ducts, K7 (fig. 3a), K8 (fig. 3b) and K18 (fig. 3c) were positive in intraductal invasion; K19 was negative (fig. 3d). To our interest, K17 was positive in some tumor cells (fig. $3 \mathrm{e}$ ). The other keratins were negative.

K7 [3], K8 [4], K18 [4] and K20 [3] are expressed in situ in MPD. However, the expression of other keratins has not been previously reported. K7, K8, K18 and K19 are expressed in the intraepidermal nests with Paget cells in our study as well as in extramammary Paget's disease (EMPD). In MPD, K7, K8, K18 and K20 are positive in Paget cells in situ in the epidermis. However, the expression of K19 has not been reported in MPD. K7, K8, K18 and K19 are positive in intraepidermal nests in MPD and in EMPD [4]. Interestingly, K17, which is a hyperproliferative keratin, was detected in intraductal invasion in the breast duct. K17 is present in basal (myoepithelial) cells in the breast duct. K17 is induced in wound healing, tumor invasion, epithelial proliferation and tumor growth [5]. The presence of K17 suggests intraductal invasion with proliferation of MPD and poor prognosis. Also, K19 was not detected in the intraductal invasion in the breast duct. Keratin expression in intraductal invasion and in situ in the epidermis in MPD may differ and vary according to the invasive pattern of tumor cells. Based on the observed pattern of keratin expression, the intraductal invasion in MPD may correlate with the presence of K17, hyperproliferative keratin and absence of K19.

Further studies on keratin expression will be made to evaluate the prognosis of MPD.

\section{Disclosure Statement}

No conflicts of interest declared. 


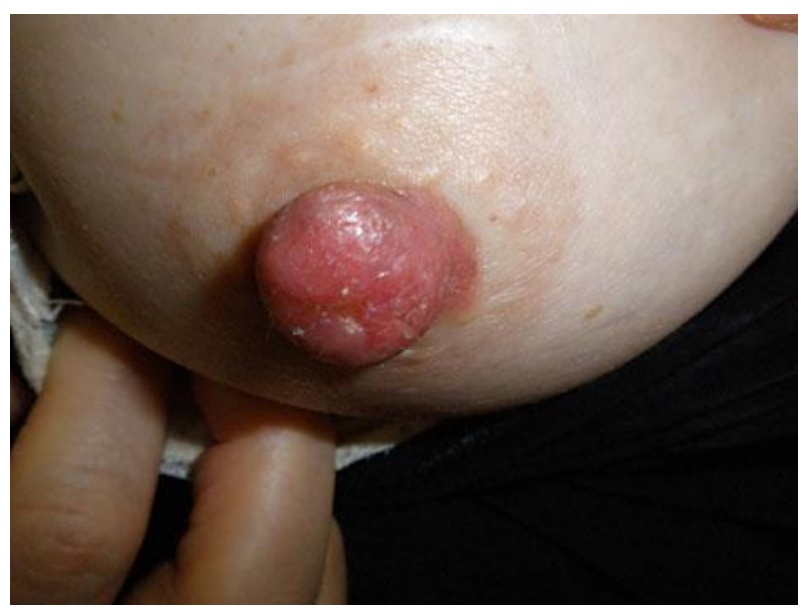

Fig. 1. Clinical findings showed erosion on the right nipple.
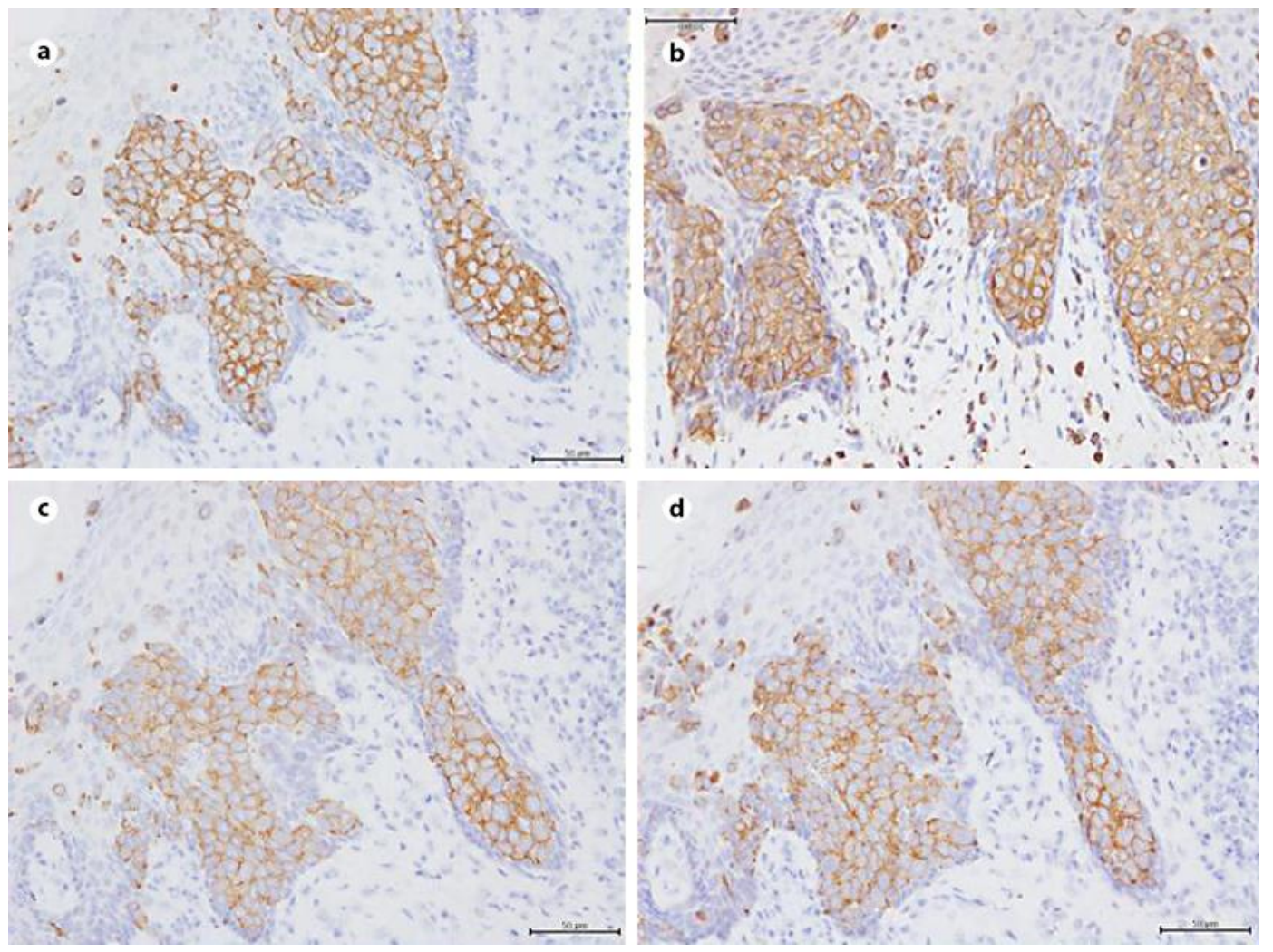

Fig. 2. Immunohistochemical staining of keratins in intraepidermal nests with Paget cells. K7 (a), K8 (b), K18 (c) and K19 (d) were expressed in the intraepidermal nests with Paget cells. 

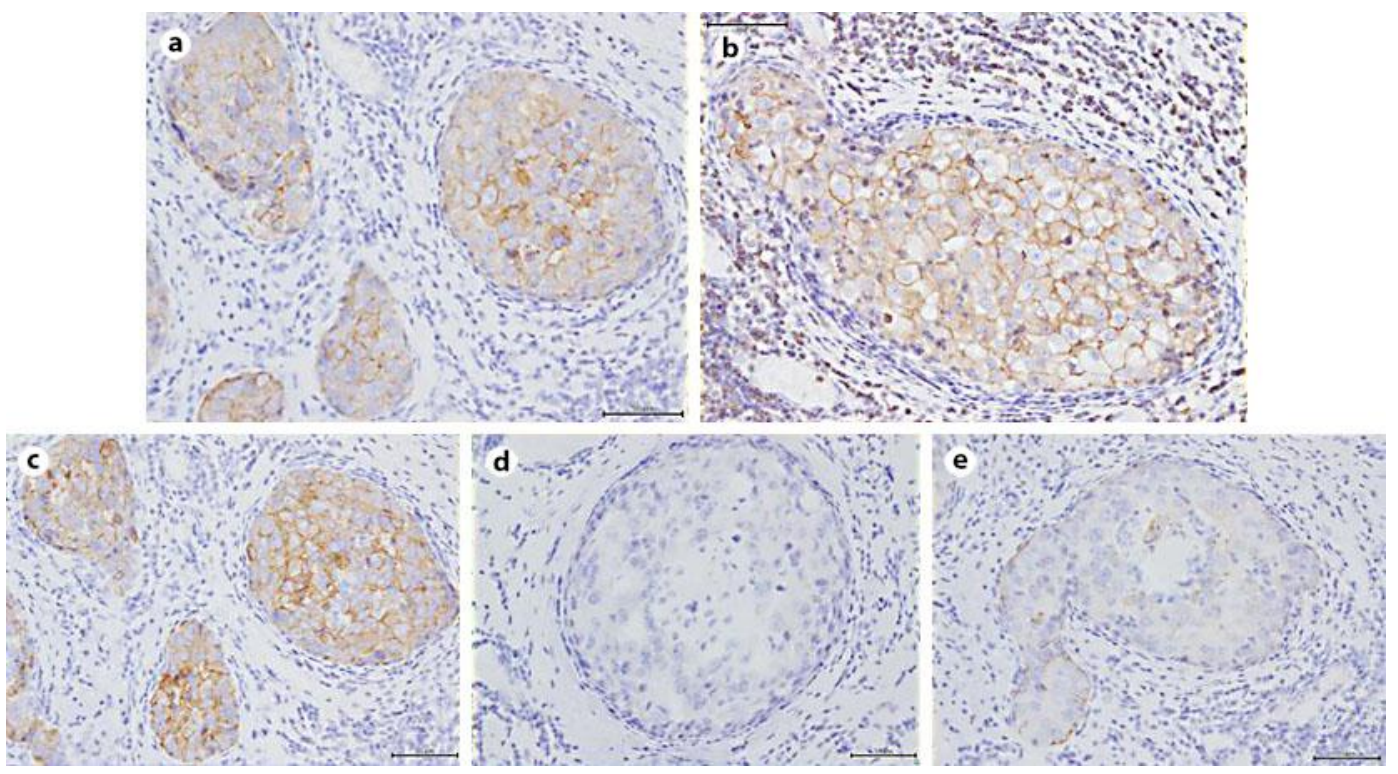

Fig. 3. Immunohistochemical staining of keratins in intraductal invasion with Paget cells. K7 (a), K8 (b), and K18 (c) were expressed in intraductal invasion, but K19 was not expressed (d). K17 was expressed in some tumor cells in intraductal invasion and myoepithelial (basal) cells (e).

\section{References}

1 Toker C: Some observations on Paget's disease of the nipple. Cancer 1961;14:653-672.

$>2$ Tsubura A, Okada H, Senzaki H, Hatano T, Morii S: Keratin expression in the normal breast and in breast carcinoma. Histopathology 1991;18:517-522.

-3 Marucci G, Betts CM, Golouh R, Peterse JL, Foschini MP, Eusebi V: Toker cells are probably precursors of Paget cell carcinoma: a morphological and ultrastructural description. Virchows Arch $2002 ; 441: 117-$ 123.

4 Liegl B, Leibl S, Gogg-Kamerer M, Tessaro B, Horn LC, Moinfar F: Mammary and extramammary Paget's disease: an immunohistochemical study of 83 cases. Histopathology 2007;50:439-447.

5 Depianto D, Kerns ML, Dlugosz AA, Coulombe PA: Keratin 17 promotes epithelial proliferation and tumor growth by polarizing the immune response in skin. Nat Genet 2010;42:910-914. 